\title{
A Suggested Theoretical Framework for Software Project Success
}

\author{
Haroon Tarawneh \\ Computer Information System, Albalqa Applied University, Salt, Jordan. \\ Email: haroontarawneh@yahoo.com \\ Received September $24^{\text {th }}, 2011$; revised October $28^{\text {th }}$, 2011; accepted November $8^{\text {th }}, 2011$.
}

\begin{abstract}
Current literature on software project management indicates that the majority of software projects either are considered a failure or challenged. These projects are characterized by exceeding budget, exceeding time, and failing to meet customer expectations. The fact that most projects fail highlights the need for research regarding the factors that lead to software project success or failure. This paper presents a theoretical framework for software project success. Based on deep analysis of current literature on software success factors, a theoretical framework is formulating. The suggested framework shows factors that have to be carefully considered in order to achieve software project success. The factors included in the framework are dividing into four categories: organizational factors, technical factors, people factors, and culture factors. The suggested framework is new in the sense that it includes many factors that are not founding together in any of the previous similar frameworks.
\end{abstract}

Keywords: Software Projects, Success Factors, Technical Factors, People Factors, Culture Factors, Organizational Factors

\section{Introduction}

According to Jones [1], many organizations indicated that a number of their software projects failed; and between one and two thirds of software projects exceed their budget and time. Further, Gibbs [2], argued that about half of the expensive software projects at the end will be considered out of control and cancelled.

In a survey of 280,000 application projects in large, medium and small cross industry companies, the Standish group showed that about $23 \%$ of projects were cancelled before completion and $49 \%$ exceeded their budgets and time scales and had fewer features and functions than originally specified [3]. Another survey by Taylor [4] showed that out of 1027 software projects studied, only 130 projects (13\%) were successful.

The Standish group [3] distinguished three types of systems: successful, challenged and impaired. A successful system means that "the system is completed on time and on budget, with all feature and functions as initially specified", a challenged system means, "a system completed and operational but over-budget, over the time estimate, and offers fewer features and function than originally specified". An impaired system means "a system that is cancelled at some point during the development cycle”.

A project is usually deemed as successful if meets requirements is delivered on time and delivered within budget [4]. Therefore software risk management is an approach that attempts to formalise risk oriented correlates of development success into a readily applicable set of principles and practise [5]. Furthermore Ropponen and Lyytinen [6] and can increase software success. Moreover the implementation of software projects is a complex task involving the successful alignment of both the technical and social system within an organization [6]. Furthermore after decades of research, systems development and implementation projects remain notoriously hard to mange and many continue to end in failure [7].

The main objective of this research is to investigate the factors that affects on software project successes/ failure of software projects in Jordanian firms.

\section{Related Work}

This section provides an overview on related work on software projects success/failure factors. The classification provided below is based on deep analysis of the literature and the authors' opinions. The software project success/failure factors are divided into four categories: 


\subsection{Organizational Factors}

The success of a software project is often related to organizational factors [8]. Organizational factors are divided into four factors: the presence of formal methodology, clear business objective, executive support and minimized project scope.

\subsection{Technical Factors}

The success of a software project is often related to technical factors [8]. Technical factors are divided into three factors: the presence of standard software infrastructure, understanding requirements and managing requirements changes and reliable estimates.

\subsection{People Factors}

The success of a software project is often related to people factors [8]. People factors are divided into two factors: user involvement and the presence of an experienced project manager.

\subsection{Culture Factor}

Denison [7] mentioned that the culture factor is a critical success factor in software project. None of these authors included this factor in their framework or models.

\section{Research Problem}

Many researchers have become interested in researching the factors that affect on software projects success/failure. Executive support affects the process and progress of project and lack of it put the project at bad situation, [911]. If project lack user involvement it fail even if it developed on time and budget, project fails if it does not meet user needs or expectation, professionals of project concern and care on this part, that lead to fail to achieve project objectives [12]. Past literatures showed that experienced software project manager can identify risk on project and lack of senior manger commitment seen as most critical risk on project $[6,11])$ Boehm and Ross [13] pointed out that the present of two different team in software development with two different objective, one deal with user requirement and another deal with technical challenges this lead to misunderstanding of objectives for the project.

For any project time is enemy for it, since scope impact time, if we minimize it we can impact it within time so the chance to success is increase [6]. In contrast to requirements which are in changeable state, infrastructure needs stability. Standish group found that about $70 \%$ of application code is infrastructure and by using standard infrastructure, the application team concentrate on business rule rather than technology standard infrastructure can shortcut applications integration that many develop- mental team fail to application it. When we create base level of requirement to our project and then develop those features we can reduce the requirement changes, help user and sponsors to see the result faster, and add benefits for project managers to prepare and link the need and criteria for the next phase of our project [14,12]. The use of good formal methodology provides realistic pictures about the project and some step may be reusable so tendency to reinvent the wheel minimized and stability of project increased, also formal methodology give manger the ability to estimate the real time so the risk is reduced [14]. In order to develop project you need to make good and realistic estimation which is hard planning and through it you purchase the requirement and component of projects, mangers must use their collective knowledge and experience to get good estimate that reflect the real effort needed. Budget and cost estimation is a crucial factor in software projects success/failure $[15,16]$. These factors include small mile-stones, proper planning, competent staff and ownership and communication skills $[6,11]$.

\section{Content Analysis}

Based on deep analysis of the above literature, the following are our findings:

1) Executive Support is an important success factor for software projects.

2) Clear Business Objective is an important success factor for software projects.

3) Formal Methodology is an important success factor for software projects.

4) Minimizing project Scope is an important success factor for software projects.

5) Standard software Infrastructure is an important success factor for software projects.

6) Understanding Requirements and Managing Requirements Changes are important success factors for software projects.

7) Reliable Estimates is an important success factor for software projects.

8) User Involvement is an important success factor for software projects.

9) Experienced Project Manager is an important success factor for software projects.

10) Culture is an important success factor for software projects as follow:

A. Culture influences the behavior of all individuals within an organization, including how decisions are made, who makes them, how rewards are given, who is promoted, how people are treated, and how the organization responds to its environment.

B. To change the culture of an organization, people need to be aware of what drives the thinking, feeling, and 
behavior of the organization.

C. Culture provides stability and predictability as it gives direction for behavior, ideas, and how to respond in various situations.

D. Information Technology manager must not only manage operations, finances, and implementation for software project but also the culture.

E. A positive relationship was found between the culture factors and other factors that affect software project success.

\section{Research Methodologies}

An empirical study as a combination of questionnaire survey and interview was applied in this research. Only 20 managers were interviewed because the others excused because of they were busy or in travelling.

\section{Research Model}

Research model in Figure 1 is built based on the combination of several past literatures. Based on these literatures the research has formed the following hypothesis: 1-There are positive relationships between (standard requirements, user involvement, executive support, clear business objective, minimized scope, reliable estimation, formal methodology, standard infrastructure, manager experience and other factors) factors And software project success.

\section{Samples}

The most of the sample are males $(n=166)$ which consist $(76.9 \%)$ of the sample where the females portion consists $(23.1 \%)$ of the sample. For education variable, bachelor degree took the high portion $(72.7 \%)$ whereas high certificates portion was (27.3\%). For experience variable, the high portion went to (11-15 years) which consisted (18.5\%); the lowest portion went to (5 years and less), (14.4\%).

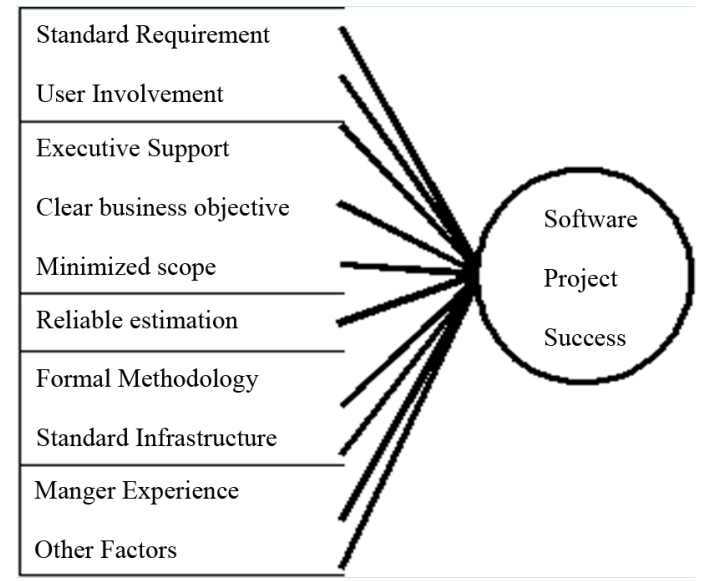

Figure 1. Research Model.

\section{Reliability Test}

Constancy factor was calculated according to (Cronbach's Alpha) for internal correspondence of total formulate and for each variable with all dimensions. The questionnaire was distributed to (25) subjects outside of the sample, the results as shown in Table 1.

The table shows that constancy factors are high and valid for statistical analysis and scientific research.

\section{Results on Factors that Contributed to Software Projects Success/Failure and the Frequency of Occurrences}

Based on the research model Figure 1, and the results that we have got from the surveys, we summarize all factors that affect on software projects success/failure according to severity degree and frequency degree.

The results show that means of influential factors in success of software engineering projects (scope, executive support, clear object, manager experience, culture, reliable estimation, standard requirement, user involvement, formal methodology, and firm infrastructure) came at high degree and the total mean of influential factors in success of software engineering projects in terms of its severity (3.63), SD (0.54); Standard requirements dimension ranked first degree with mean reached(3.96) followed by user involvement (3.89), executive support at third degree (3.67), clear objective at fourth degree (3.64), while the dimension of other factors ranked tenth rank with mean (3.42) at middle degree.

Table 1. Value of internal correspondence for each variable (independent and dependent) with all dimensions.

\begin{tabular}{cl}
\hline Variable and dimensions & Constancy factor \\
\hline All independent variables & 0.91 \\
Standard requirement & 0.86 \\
User involvement & 0.85 \\
Executive support & 0.88 \\
Clear objectives & 0.90 \\
Scope & 0.84 \\
Reliable estimation & 0.81 \\
Formal methodology & 0.86 \\
Firm infrastructure & 0.83 \\
Manager experience & 0.85 \\
Other factors & 0.78 \\
Dependent variable & 0.85 \\
Total( variables, dimensions and items) & 0.89 \\
\hline
\end{tabular}


Also the results show that means of influential factors in success of software engineering projects ( scope, executive support, clear object, manager experience, culture, reliable estimation, standard requirement, user involvement, formal methodology, and firm infrastructure) came at high degree and the total mean of influential factors in success of software engineering projects in terms of its frequency (3.56), SD (0.57); culture dimension ranked first degree with mean reached(3.87) followed by standard requirement (3.81), user involvement at third degree (3.59), executive support at fourth degree (3.58), while the dimension of manager experience ranked tenth rank with mean (3.34) at middle degree.

\section{Result Discussions}

\subsection{Standard Requirement Factor}

This factor came at first rank in terms of severity degree and frequency. From the researcher's view, weakness of workers in collecting requirements and disability to use the right methods in collection process is from the reasons that lead to make these requirements unclear. Each project has its privacy in requirements collection process by the user and analyzing these requirements; also if these requirements don't identify accurately, this may cause failure to the project; documentation mechanism considered as an important requirement that is through revising available documents in Jordanian institutions there was no documentation mechanism for requirements in order to revise it with systems users when finishing these systems at the end. User's misunderstanding of needed requirements is one of the reasons that lead to imperfection of requirements and changing them during working on the system. Identifying project framework, clarifying and defining objective, and users involvement play an important role in defining the project requirements.

\subsection{User Involvement Factor}

This factor ranked the second rank in terms of severity and frequency degree to assure the interest in continuation of teamwork members when they doing their works perfectly without any imperfection may affect negatively quantities and qualitative outcomes. For the importance of the big role that user involvement plays in success of software engineering projects, this dimension is very important to clarifying work objectives and making balance among teamwork members when they sharing their roles and enable them to know unclear things in the institution, so, involving the user within teamwork helps in resolving some of vagueness in specific items of the work which is to be achieved by software engineering team in the institution. User involvement process minimizing resistance change for new system that is the user who intended to be involved within teamwork should have a positive role to protect the project and tries to convince the others with the importance of the project for the institution in all; also this user should have a role in communication flexibility process between system development team and system users because he/she is the only one who can explain the teamwork and his /her coworkers views.

\subsection{Executive Support Factor}

In terms of severity and frequency degree in success of software engineering projects, this dimension ranked the third rank. Executive administration will minimize difficulties that facing system development team. Support will be provided to the project if the expected benefits are big and if the project can be applied in more than one location in the institution. Software engineering manager should have the ability to attract executive administration in order to provision support and distributing the available resources to the project's phases.

\subsection{Clear Business Objective Factor}

In terms of severity and frequency degree in success of software engineering projects, this dimension ranked the fourth rank. If the objective is clear, this will facilitates teamwork task and identifying the project requirement early as well as that will play an important role in defining the scope framework; here, the software engineering manager and his teamwork have to define the real objecttives that are put by the administration, also holding successive meeting with administration in order to clarifying the required objectives to facilitate controlling the work's phases. By doing this, the project manager is able to revise the plan that is put to achieve the required objective and enable him to know the inadequacy in the plan which helps in avoiding failure of the project.

\subsection{Minimized Scope Factor}

This factor came at fifth rank in terms of severity and frequency. If the size of work has been minimized and divides into phases, this will contributes to success of the software engineering projects. Project's size affects the required time to accomplish the project and the estimated budget of the project. Furthermore, minimizing the project size will affect the formal methodology of the project.

\subsection{Reliable Estimation Factor}

This factor came at sixth rank in terms of severity and frequency. Reliable estimation is very important to success of software engineering, but in Jordanian environment there is a lack of trained people who are able to use the estimation tools, instead of that they depend on esti- 
mations and experiences of the project manager in putting the estimated cost of the costs and time as well as expected benefits that could be gained from project; this may involve risk because each project has its special environment, so changing factor in requirements may make the project exceed the estimated budget and minimized scope that this factor affect the reliable estimation. Finally, the technological development and the short age of this technology make the project to exceed the estimated budget.

\subsection{Formal Methodology Factor}

This dimension came at seventh rank in terms of severity and frequency. If there is a formal methodology of the software engineering projects and is used in all phases of the project, this well lead to success of the project and helps the manager to control the project. A clear template should be used during all talks that take place between teamwork and the user and between the manager and teamwork; this communication helps the manager to revise this template to identify the mistake, change and any delay in the project in order to avoid them to protect the project from failure.

\subsection{Standard Software Infrastructure Factor}

This factor came at eighth rank in terms of severity and frequency. Existing this dimension will help in success of the project. The results indicated that the good infrastructure is available in Jordanian environment which may help in developing software engineering projects through the policies that are put by governmental and private institutions in Jordan, but the changeable technology and the short period of technology affect the institutions to follow up these developments especially hardware. From the researcher's view, the institutions that have culture supports computerization, they will seek to provide the right infrastructure for software engineering projects and be able to follow up the digital age that we live in.

\subsection{Manager Experience Factor}

This factor took the ninth rank and at middle degree according to the scale that was used by the study, this indicates that the sample individuals have the ability to work and solving problems within teamwork spirit. Although, manager experience has an important role in success of engineering software, the results indicated that there were significant differences at subjects' perceptions towards the effective factors in success of software engineering projects that attributed to experience variable; the differences were in favorers to subjects whose experience more than 21 years.

\subsection{Other Factors}

This study showed that ineffective communication was one of the main factors for project failure. Unrealistic planning for software project lead to project failure. study done by Addison and Keil et al. concern on the realistic planning and good communication skill to software project success.

\section{Theoretical Framework for Software Project Success}

Based on deep analysis of the literature and our findings, we suggest the following theoretical framework for software project success (see Figure 2). We believe that Software project management is a lengthy undertaking involving a set of complex activities that take a lot of time and cost. The success in such an undertaking depends on good and reliable estimation. Based on the above analysis, we classify software project success factors into four categories: organizational factors which are the presence of formal methodology, clear business objective, executive support and project scope, technical factors that are standard software infrastructure, understanding requirements and managing requirements changes and reliable estimates, people factors that are user involvement and experienced project manager, and the cultural factors which involve organizational culture. Also in the analysis, a relationship was found between the organizational factors, people factors, technical factors.

\section{Conclusions}

The research attempts to understand the factors that affect

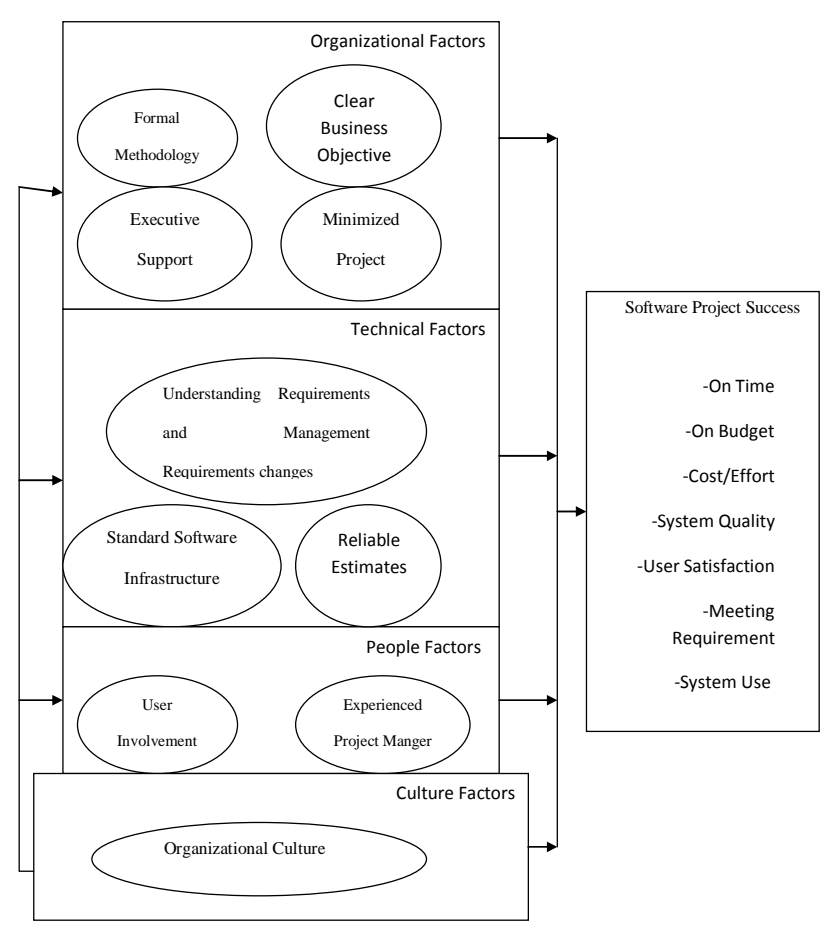

Figure 2. The suggested framework. 
software projects success. Furthermore, this paper attempts to understand the cultural influences on software projects and the interaction between the culture factor and other factors that affect software project success. In addition, this paper provides the interested audience, either in private or academic sectors, with a short description of those factors that result in software project success/failure. Finally in this paper, a theoretical framework is suggested.

The deep analysis of the literature showed that project success is dependent on many factors including executive support, clear objectives, presence of a formal methodology, minimization of project scope, use of a standard software infrastructure, understanding and managing requirements, making reliable estimates, user involvement, presence of experienced project manager, and last not least, taking cultural aspects into consideration. The work presented above resulted in the formation of a theoretical framework for software project success. This framework is novel in the sense that it includes many factors that are not found together in any other framework. The framework stresses the importance of cultural factors in project success. The factors included in the framework are validated through content analysis of the literature. The suggested framework is currently being calibrated by using it to evaluate many ongoing and completed software projects. The results will be presented in a forthcoming paper.

\section{REFERENCES}

[1] C. Jones, “Applied Software Measurement: Assuring, Productivity and Quality,” 2nd Edition, McGraw-Hill, New York, 1997.

[2] W. Gibbs, "Software's Chronic Crisis," Scientific American, September, Vol. 167, No. 4, 1999, pp. 468-473.

[3] Standish group, extreme chaos, 2001, Standish group international. http://www.standishgroup.com

[4] L. J. May, "Major Causes of Software Project Failures," 2002. http://stsc.hill.af.mil/crosstalk/1998/jul/causes.asp
[5] A. Taylor, "IT Projects: Sink or Swim,” The Computer Bulletin, 2000, pp. 24-26. doi:10.1093/combul/42.1.24

[6] J. Ropponen and K. Lyytinen, "Components of Software Development Risk: How to Address Them?” IEEE Transactions on Software Engineering, Vol. 26, No. 2, 2000, pp. 98-111. doi:10.1109/32.841112

[7] D. R. Denison, Corporate Culture and Organizational Effectiveness, John Wiley \& Sons, New York, 1990.

[8] F. J. Heemstra and R. J. Kusters, "Dealing with Risk: A Practical Approach,” Journal of Information Technology, Vol. 11, 1996, pp. 333-346. doi:10.1057/jit.1996.7

[9] D. Radcliffe, "Project Leadership," Software Magazine, Vol. 18, No. 5, 1998, pp. 38-44.

[10] N. H. Arshad, A. Mohamed and Z. Matnor, "Software Development Projects: Risk Factors and Occurrences," WSEAS Transactions on Computer Research, Vol. 2, No. 2, 2007, pp. 1991-8755.

[11] T. Addison and S. Vallabh, "Controlling Software Project Risks-An Empirical Study of Methods Used by Experienced Project Mangers,” Proceedings of SAICSIT, 2002, pp. 128-140.

[12] A. Mursu, H. A. Soriyan and K. C. Olufokunbi, “Toward Successful ISD in Developing Countries: First Results from a Nigerian Risk Study Using the Delphi Method," 1996.

[13] B. Boehm and R. Ross, "Theory-W Software Project Management Principles and Examples,” IEEE Transactions on Software Engineering, Vol. 15, No. 7, 1989, pp. 902-916. doi:10.1109/32.29489

[14] E. W. Martin, D. W. Dehayes, J. A. Hoffer and W. C. Perking, "Managing Information Technology: What Mangers Need to Know," $2^{\text {nd }}$ Edition, Prentice Hall, New Jersey, 1994.

[15] B. Farbey, F. Land and D. Targett, "Moving IS Evaluation Forward: Learning Themes and Research Issues," Journal of Strategic Information Systems, Vol. 8, 1999, pp. 189-207. doi:10.1016/S0963-8687(99)00021-9

[16] T. Eldabi, R. J. Paul and H. Sbeih, "Operational Use Evaluation/Post Implementation Evaluation of IT,” In: M. Levy, A. Martin and C. Schweighart, Eds., Proceedings of UKAIS, 9-11 April, Warwick University, Warwick, 2003. 